\title{
ÁCIDO ABSCÍSICO E ETEFOM: INFLUÊNCIA SOBRE A MATURAÇÃO E QUALIDADE DAS UVAS CABERNET SAUVIGNON ${ }^{1}$
}

\author{
JOÃO PETERSON PEREIRA GARDIN² ${ }^{2}$ RAFAEL LIZANDRO SCHUMACHER ${ }^{3}$, \\ JEAN CARLOS BETTONI ${ }^{4}$, JOSÉ LUIZ PETRI ${ }^{5}$, EDSON LUIZ DE SOUZA ${ }^{6}$
}

RESUMO - Neste estudo, objetivou-se determinar os efeitos da aplicação exógena de ABA e ETEFOM sobre a maturação e a qualidade de uvas Cabernet Sauvignon. O presente trabalho foi realizado em um vinhedo, localizado na Estação Experimental da Epagri de Videira - SC, durante a safra de 2009/2010. Foram utilizadas as doses de $0 ; 20$ e 40g i.a. $100 \mathrm{~L}^{-1}$ de ácido abscísico (ABA) e 0; 136,8 e 273,6g i.a. 100L $\mathrm{L}^{-1}$ de ETEFOM, configurando um experimento fatorial $3 \times 3$. Os tratamentos foram aplicados no início da mudança de cor das bagas "veraison". As variáveis analisadas nas uvas foram teor de antocianinas, polifenóis totais, índice CIRG (índice de cor para uvas tintas), atividade antioxidante, sólidos solúveis totais ( ${ }^{\circ}$ Brix) e acidez total titulável. Os resultados indicam melhoria na qualidade das uvas com o uso das combinações de ABA e ETEFOM, aumento do índice CIRG, polifenóis totais, antocianinas e açúcares.

Termos para indexação: Vitis vinifera L., reguladores de crescimento, coloração das bagas, antocianinas, polifenóis totais.

\section{ABSCISIC ACID AND ETEFOM: INFLUENCE ON THE MATURITY AND QUALITY OF CABERNET SAUVIGNON GRAPES}

\begin{abstract}
This study aimed to determine the effects of exogenous application of ABA and ETEFOM on the maturation and quality of Cabernet Sauvignon. This study was performed in a vineyard, located at the Experimental Station of Videira - Epagri - SC, during the 2009/2010 harvest. The doses of 0, 20, 40 g i.a.100L-1 of the abscisic acid (ABA) and 0, 136.8 and 273.6 g i.a. 100L-1 of ETEFOM, forming a $3 \times 3$ factorial design. The treatments were applied at the beginning of the color change 'veraison'. The variables were analyzed in grapes anthocyanins, total polyphenol, CIRG index (color index for red grapes), antioxidant activity, total soluble solids ( $\left.{ }^{\circ} \mathrm{Brix}\right)$ and titratable acidity. The results show improvement in the quality of grapes with the use of ABA and ETEFOM combinations, increased CIRG index, polyphenols, anthocyanins and sugars.
\end{abstract}

Index terms: Vitis vinifera L., growth regulators, color berries, anthocyanins, total polyphenols.

\section{INTRODUÇÃO}

A vitivinicultura brasileira vem evoluindo nos últimos anos em busca de novas tecnologias que introduzam melhorias na qualidade do vinho, tornando-o competitivo no cenário internacional. Inúmeras são as dificuldades brasileiras na produção de vinhos de qualidade, principalmente as relacionadas com fatores ambientais, tais como solo e clima. $\mathrm{Na}$ maioria dos anos, a alta incidência de chuvas e a baixa amplitude térmica durante a maturação das uvas fazem com que elas não alcancem índices ótimos de maturação.
A Epagri de Videira difundiu aos agricultores da região um sistema que usa cobertura plástica para a proteção da parreira contra a umidade excessiva na folha, proporcionada pela alta precipitação. A diminuição da umidade nas folhas promove a diminuição do aparecimento de doenças fúngicas; no entanto, os agricultores têm percebido a diminuição na coloração de uvas tintas com o uso de cobertura plástica.

O uso de reguladores de crescimento na maturação das uvas vem sendo empregado em vários países. Alguns estudos demonstram que aplicações externas de ABA (ácido abscísico) e ETEFOM (áci-

\footnotetext{
(Trabalho 210-11). Recebido em: 24-08-2011. Aceito para publicação em: 14-04-2012.

${ }^{2}$ Eng. Agr., Dr., Epagri - Estação Experimental de Videira. Rua João Zardo, 1660, CEP 89560-000, Videira-SC. E-mail: joaogardin@epagri.sc.gov.br

${ }^{3}$ Eng. Agrônomo, Doutorando em Enologia - Universidad Castilla La-Mancha, Espanha, Bolsista Embrapa Clima Temperado. E-mail: rlschumacher@gmail.com

${ }^{4}$ Eng. Agrônomo, bolsista da Embrapa Clima Temperado. E-mail: jcbettoni@gmail.com

${ }^{5}$ Eng. Agr., MSc., Epagri - Estação Experimental de Caçador, CEP 89500-000, Caçador-SC. E-mail: petri@epagri.sc.gov.br

${ }^{6}$ Eng. Agr., Dr., Epagri - Est. Exp. de Videira. Rua João Zardo, 1660, CEP 89560-000, Videira-SC. E-mail: edsonluiz@epagri.sc.gov.br
} 
do 2-cloroetil fosfônico), por exemplo, antecipam a época de colheita e aumentam as concentrações de antocianinas e proantocianidinas na casca das uvas tratadas, melhorando consideravelmente sua coloração, originando uvas com uma maturação mais uniforme e de melhor qualidade (CANTÍN et al., 2007; LACAMPAGNE et al., 2010).

A coloração das bagas de uvas tintas é produzida por um grupo de antocianinas e proporciona características importantes na elaboração de vinhos tintos de qualidade. $\mathrm{O}$ acúmulo de antocianinas parece estar regulado, ao menos em parte, pelo ABA (HIRATSUKA et al., 2001; LACAMPAGNE et al., 2010), sendo que as aplicações exógenas desse hormônio, além de aumentar as concentrações de antocianinas nas cascas das uvas (PEPPI et al., 2006), também antecipam o desenvolvimento da coloração em comparação com as uvas não tratadas (HIRATSUKA et al., 2001; PEPPI et al., 2006).

Do mesmo modo, o ETEFOM tem sido utilizado com sucesso em muitas cultivares de Vitis vinifera L., principalmente por acentuar a coloração das bagas, diminuir a acidez e aumentar indiretamente a relação açúcar/acidez (DELGADO et al., 2004). El-Kereamy et al. (2003) demonstraram um aumento da expressão gênica de enzimas envolvidas na biossíntese de antocianinas, tais como UDP-flavonoide glicosiltrasferase (UFGT), com concomitante aumento no acúmulo de antocianinas em Vitis vinifera L. cv. Cabernet Sauvignon.

Neste estudo, objetivou-se determinar os efeitos da aplicação exógena de ABA e ETEFOM sobre a coloração das bagas, concentração de antocianinas e polifenóis, açúcares, acidez e atividade antioxidante nas uvas da variedade Cabernet Sauvignon tratadas no início da maturação.

\section{MATERIAL E MÉTODOS}

O experimento foi realizado na safra de 2009/2010, em um vinhedo de oito anos composto pela cv. Cabernet Sauvignon, conduzido em sistema "Y" (tipo manjedoura), com porta-enxerto Paulsen 1103 (Vitis berlandieri x Vitis rupestris) e sob cobertura plástica, localizado no campo experimental da Epagri de Videira - SC. O delineamento experimental foi o inteiramente casualizado, com cinco repetições (plantas) e os tratamentos dispostos em esquema fatorial $3 \times 3$, com nove tratamentos, que são as combinações possíveis entre os três níveis do fator $\mathrm{ABA}$ e os três níveis do fator ETEFOM, conforme descrito a seguir: T1- $0+0$ g i.a. $100 \mathrm{~L}^{-1}$ de $\mathrm{ABA}+\mathrm{ETEFOM}$, respectivamente; T2- $0+136,8 \mathrm{~g}$ i.a. $100 \mathrm{~L}^{-1}$ de $\mathrm{ABA}+\mathrm{ETEFOM}$; T3- $0+273,6$ g i.a.
100L $\mathrm{L}^{-1}$ de ABA + ETEFOM; T4- $20+0$ g i.a. $100 \mathrm{~L}^{-1}$ de ABA + ETEFOM; T5- $20+136,8$ g i.a. $100 \mathrm{~L}^{-1} \mathrm{de}$ ABA + ETEFOM; T6- $20+273,6$ g i.a. $100 \mathrm{~L}^{-1}$ de ABA + ETEFOM; T7- $40+0$ g i.a. $100 \mathrm{~L}^{-1}$ de ABA + ETEFOM; T8- $40+136,8$ g i.a. $100 \mathrm{~L}^{-1}$ de ABA + ETEFOM, e T9- $40+273,6$ g i.a. $100 \mathrm{~L}-1$ de ABA + ETEFOM. As aplicações dos produtos foram realizadas no estádio de mudança de cor da casca "veraison", o volume de calda foi de 1.000 L.ha $^{-1} \mathrm{e}$ foi usado espalhante adesivo não iônico na dose recomendada pelo fabricante. Os dados foram submetidos à análise de variância, e as médias, comparadas pelo teste de Tukey, a 5\% de probabilidade de erro, realizadas com auxílio do aplicativo R Development Core Team (2011).

Foram coletadas amostras (cachos) aleatoriamente, de cada uma das cinco plantas (repetições) para a realização das análises bioquímicas. Para a extração dos compostos e a análise de polifenóis, antocianinas e atividade antioxidante, foram pesados $100 \mathrm{~g}$ de casca de uva e adicionados $40 \mathrm{~mL}$ de metanol a $50 \%$ e colocado em estufa a $30^{\circ} \mathrm{C}$, por 24 horas. Retirou-se o sobrenadante e reservou-se em frasco hermeticamente fechado. As cascas foram ressuspendidas em metanol a $50 \%$ e colocadas em refrigerador a $-15^{\circ} \mathrm{C}$, por 24 horas. Após esse período, o sobrenadante foi coletado e misturado com o extraído anteriormente. Com mais $20 \mathrm{~mL}$ de metanol a $50 \%$, foi realizada a lavagem das cascas, e o extrato foi misturado aos anteriores, totalizando $100 \mathrm{~mL}$ de solvente por $100 \mathrm{~g}$ de casca de uva. O extrato foi mantido em geladeira até o momento da análise.

A concentração de polifenóis totais foi determinada por espectrofotometria, de acordo com o método colorimétrico de Folin-Ciocalteu, descrito por Waterhouse (2001), e o resultado, expresso em mg. $\mathrm{L}^{-1}$ de ácido gálico. A atividade antioxidante foi avaliada utilizando o método do sequestro de radicais livres do DPPH (2,2-difenil-1-picrilhidrazil) (ALMEIDA et al., 2006), e a unidade de medida é porcentagem de sequestro de radicais livres DPPH (\%SRL). A determinação de antocianinas totais fundamenta-se no $\mathrm{pH}$ diferencial, em que a estrutura da molécula de antocianina é modificada pelo $\mathrm{pH}$, sendo a leitura no espectrofotômetro feita a $520 \mathrm{~nm}$, e os resultados, expressos em mg. $\mathrm{L}^{-1}$ de antocianinas, sendo o padrão usado a malvidina 3-glicosídeo. As análises de ${ }^{\circ}$ Brix e acidez total foram feitas através dos métodos descritos por Rizzon (2010).

A coloração das bagas das uvas foi determinada com auxílio de um colorímetro Minolta ${ }^{\circledR}$, modelo CR-300, com fonte de luz D 65, e com 8 $\mathrm{mm}$ de abertura. Os valores são expressos na coordenada-padrão CIE L* a* b*, em que $L^{*}$ representa 
o brilho de uma superfície $\left(L^{*}=100=\right.$ branco; $L^{*}=0$ $=$ preto); $\mathrm{a}^{*}$ representa a intensidade de cor do verde ao vermelho $\left(a^{*}\right.$ mais negativo $=$ mais verde; $a^{*}$ mais positivo $=$ mais vermelho), e $b^{*}$ mede a intensidade de cor do amarelo ao azul $\left(b^{*}=\right.$ mais positivo $=$ mais amarelo; $b^{*}=$ mais negativo $=$ mais azul). Os valores de $a^{*} \mathrm{e} b^{*}$ foram utilizados para calcular a tonalidade de cor $C^{*}$, pela fórmula $\mathrm{C}^{*}=\left(\mathrm{a}^{* 2}+\mathrm{b}^{* 2}\right)^{1 / 2} \mathrm{e} \mathrm{o}$ ângulo $\mathrm{H}^{*}$. Embora muitas bagas apresentassem diferenças visuais na coloração, estas eram impossíveis de serem expressas numericamente (CARREÑO et al., 1995). Por isso, foi calculado o índice específico para bagas de cor vermelha, CIRG (color index for red grapes), a partir de valores da colorimetria, com a equação $\mathrm{CIRG}=180$-hue $*\left(\mathrm{~L}^{*}+\mathrm{C}^{*}\right)$, em que o hue* é o ângulo calculado através da seguinte fórmula hue* $=$ Tang${ }^{1}\left(b^{*} / a^{*}\right)$. Valores do índice CIRG próximos de zero correspondem a bagas de coloração verde, enquanto valores próximos de três correspondem a bagas de coloração vermelha (CARREÑO et al., 1995). Foram avaliadas 50 bagas por repetição, sendo coletadas ao acaso, no dia da colheita dos cachos utilizados para as análises bioquímicas.

\section{RESULTADOS E DISCUSSÃO}

As condições climáticas durante o período experimental são apresentadas na Tabela 1 . As temperaturas foram consideradas normais para a região quando comparadas com as médias dos últimos 40 anos. Já as precipitações pluviais estão um pouco acima da média, fator que pode prejudicar a maturação das bagas, principalmente pela diminuição da radiação solar, diminuição que ocorre também com o uso da cobertura plástica. Segundo Rocha e Guerra (2008), a luz solar é o fator climático preponderante para o desenvolvimento da baga da uva, uma vez que influencia na composição da fruta através de enzimas relacionadas à síntese dos compostos como a fenilalanina amonioliase - PAL, enzima-chave na síntese dos compostos fenólicos. Kolb et al. (2003) relatam que a síntese de polifenóis foi estimulada por luz natural, principalmente UV-B, ao trabalharem em condições controladas em casa de vegetação. A cobertura plástica possivelmente reduz a exposição da planta à radiação UV-B, pois a cobertura sofre tratamento para esse tipo de comprimento de onda que a danifica e diminui sua durabilidade. Já Gardin et al. (2010) relatam que a cobertura plástica aumenta o conteúdo de sólidos solúveis totais e diminui a acidez total titulável, o que é bom para as condições de cultivo do Sul do Brasil.

Na Tabela 2, observa-se o efeito do ABA e do ETEFOM na coloração das bagas das uvas pela quantificação do índice CIRG. Essa avaliação demonstrou que houve efeito significativo do ABA, o qual promoveu aumento do grau de maturação da uva, indicado pelo aumento do índice CIRG com o aumento da concentração aplicada. Esses resultados demonstram que o ABA exerce efeito na maturação das uvas, principalmente por modificar sua coloração. O ETEFOM influenciou no amadurecimento das bagas com o aumento da concentração aplicada, exceto quando não combinado com $\mathrm{ABA}$. Esse efeito está de acordo com o encontrado no conteúdo de polifenóis (Tabela 4). Cantín et al. (2007) demonstraram que a aplicação de $150 \mu \mathrm{L}$ e $300 \mu \mathrm{L}$ de ABA, no início da maturação das uvas Crimson Seedless (Vitis vinifera L.), antecipou a época de colheita em 10 e 30 dias, respectivamente, em comparação com as uvas tratadas com ETEFOM ou não tratadas. Já Hiratsuka et al. (2001) comprovaram que maiores níveis de pigmentação nas cascas das uvas tratadas com ABA foram acompanhadas por maior atividade da CHFI (chalcona-flavanona isomerase). A atividade da CHFI está estreitamente relacionada com a biossíntese de antocianinas. Esta relação pode ser corroborada, a seguir, pela análise de antocianinas e polifenóis nas bagas.

Na Tabela 3, observa-se o incremento dos teores de antocianinas nas uvas de acordo com o aumento das concentrações de ABA e ETEFOM, ocorrendo interação significativa entre esses fatores. Isso se deve, principalmente, a que o ABA atua diretamente na síntese de antocianinas. Colli e Purgatt (2008) demonstram que o ABA pode atuar como promotor da síntese de etileno, o que explicaria o maior desenvolvimento da pigmentação e a maior concentração de antocianinas nessas uvas, além do adiantamento da maturação.

Na Tabela 4, é demonstrado o efeito sobre a concentração de polifenóis totais nas uvas. Foi verificada a interação dos fatores, o que promoveu um efeito de ABA e ETEFOM no aumento do conteúdo de polifenóis totais. Isso se deve, do mesmo modo que na produção de antocianinas, ao fato de o ABA e de o ETEFOM estarem relacionados com a biossíntese desses compostos. Lacampagne et al. (2010), indicam que o ABA é um corregulador da leucoantocianidina redutase (LAR) e da antocianidina redutase (ANR), ambas responsáveis pela formação de (+)-catequinas e (-)-epicatequinas nas uvas.

Na Tabela 5, é apresentada a atividade antioxidante das uvas, a qual indica um aumento dessa atividade nas bagas tratadas com ABA e ETEFOM. Esses resultados podem ser explicados pelos maiores 
índices de compostos fenólicos encontrados nos mesmos tratamentos (Tabelas 3 e 4). O conteúdo de polifenóis e antocianinas está correlacionado positivamente com a atividade antioxidante, conforme Soares et al. (2008), que trabalharam com as cultivares Niágara Rosada e Isabel. O aumento do conteúdo desses compostos melhora a qualidade da uva e contribui para a saúde dos consumidores dos produtos oriundos de uvas com maior atividade antioxidante, pois combatem os radicais livres tão prejudiciais ao nosso organismo.

Na Tabela 6, são apresentados os resultados de conteúdo de sólidos solúveis totais ( $\left.{ }^{\circ} \mathrm{Brix}\right)$. Observa-se que, na ausência de ETEFOM, a aplicação de 20 ou 40 g i.a. $100 \mathrm{~L}^{-1}$ aumentou o grau Brix em mais de 0,8 , o que é um valor considerável do ponto de vista enológico. O mesmo aconteceu para ABA dentro de ETEFOM 136,8, enquanto na concentração mais alta de ETEFOM (273,6 g i.a. 100L $\left.\mathrm{L}^{-1}\right)$ o ABA não apresentou diferença no conteúdo de sólidos solúveis totais. Já o ETEFOM, na dose de 273,6 g i.a. $100 \mathrm{~L}^{-1}$, apresentou aumento do conteúdo de sólidos solúveis totais quando na ausência de $\mathrm{ABA}$ e na dose de $136,8 \mathrm{~g}$ i.a. $100 \mathrm{~L}^{-1}$, quando na presença de $\mathrm{ABA}$ na dose de $20 \mathrm{~g}$ i.a. $100 \mathrm{~L}^{-1}$. Já na dose de 40 g i.a. $100 \mathrm{~L}^{-1}$ de $\mathrm{ABA}$, o ETEFOM não apresentou diferença significativa. Pereira e Fracaro (2004), estudando a cultivar Niágara Rosada, não encontraram diferença significativa no teor de sólidos solúveis ao aplicarem doses de 0;3;6 e 9 L.ha $^{-1}$ de ETEFOM, ou seja, doses até três vezes mais altas que as usadas neste trabalho. Da mesma forma, Rodrigues et al. (2010) não encontraram diferenças nos sólidos solúveis aplicando as doses de $0 ; 120 ; 240$; 360; 480; 720 e 960 mg.L.-1 de ETEFOM, por imersão dos cachos no início da mudança da coloração. Essas concentrações utilizadas chegam, na dose mais alta, a três vezes a utilizada no presente manuscrito. Isso indica que o aumento dos sólidos solúveis se deve mais à presença do ABA do que do ETEFOM; no entanto, o uso de ABA para esse fim deve ser ainda mais estudado.

Na Tabela 7, são apresentados os resultados para a variável acidez titulável e pode-se observar que o coeficiente de variação $(\mathrm{CV})$ foi muito baixo, possibilitando identificar pequenas diferenças entre os tratamentos. No entanto, do ponto de vista enológico, essas diferenças não são significativas. Verificou-se um aumento da acidez na dose de 136,8 g i.a. $100 \mathrm{~L}^{-1}$ de ETEFOM, quando combinada com $0 ; 20$ ou $40 \mathrm{~g}$ i.a. $100 \mathrm{~L}^{-1}$ de $\mathrm{ABA}$; no entanto, nas doses mais altas de ETEFOM $(273,6)$, a acidez foi mais baixa que na dose zero, quando combinada com as doses de $20 \mathrm{e}$ $40 \mathrm{~g}$ i.a. $100 \mathrm{~L}^{-1}$ de ABA. Isso indica que a acidez tem seu controle mais intenso com outros fatores que não os reguladores ETEFOM e ABA.

TABELA 1 - Condições climáticas durante o período experimental e em comparação com as médias dos últimos 40 anos. Videira-SC, 2010.

\begin{tabular}{lccccccc}
\hline \multicolumn{3}{c}{ Período Experimental } & \multicolumn{4}{c}{ Média últimos 40 anos } \\
\hline Meses & $\mathrm{T}^{\mathrm{o}}$ Máx.* & $\mathrm{T}^{\mathrm{o}}$ Min.** & P.P.*** & Meses & $\mathrm{T}^{\mathrm{o}}$ Máx.** & $\mathrm{T}^{\mathrm{o}}$ Mín.** & P.P.*** \\
\hline Outubro 2009 & 25,5 & 13,6 & 157,6 & Outubro & 24,7 & 12,8 & 205,3 \\
Novembro 2009 & 29,5 & 17,8 & 270,5 & Novembro & 26,6 & 13,9 & 170,2 \\
Dezembro 2009 & 29,2 & 17,9 & 140,7 & Dezembro & 27,9 & 15,9 & 162,4 \\
Janeiro 2010 & 28,8 & 18,4 & 282,2 & Janeiro & 28,3 & 17 & 187 \\
Fevereiro 2010 & 29,9 & 18,3 & 242,8 & Fevereiro & 28,4 & 16,8 & 172,3 \\
Março 2010 & 26,9 & 15,7 & 268,5 & Março & 27,6 & 15,5 & 131,3 \\
\hline média & 28,30 & 16,95 & 227,05 & média & 27,25 & 15,32 & 171,42 \\
* temperatura máxima; ** temperatura mínina; *** precipitação pluvial. & & & &
\end{tabular}


TABELA 2 - Efeito das aplicações de ABA e ETEFOM no índice de coloração para uvas tintas (CIRG - color index for red grape) de uvas Cabernet Sauvignon sob cobertura plástica. Videira-SC, 2010.

\begin{tabular}{ccccc}
\hline & & \multicolumn{3}{c}{ ETEFOM (g i.a. 100L $\left.\mathrm{L}^{-1}\right)$} \\
\cline { 2 - 5 } & 0 & $1,57 \mathrm{bA}$ & 136,8 & 273,6 \\
\hline \multirow{2}{*}{ ABA } & 20 & $1,65 \mathrm{bB}$ & $1,71 \mathrm{bAB}$ & $1,52 \mathrm{bA}$ \\
$\left(\right.$ g i.a. $\left.100 \mathrm{~L}^{-1}\right)$ & 40 & $2,00 \mathrm{aA}$ & $2,06 \mathrm{aA}$ & $1,90 \mathrm{aA}$ \\
& \multicolumn{4}{c}{ CV 38,75\% } \\
\hline
\end{tabular}

Letras minúsculas iguais (na coluna) e maiúsculas iguais (na linha) não diferem ao nível de $5 \%$ de probabilidade de erro, pelo teste de Tukey.

TABELA 3 - Efeito das aplicações de ABA e ETEFOM na concentração de antocianinas de cascas de uvas Cabernet Sauvignon sob cobertura plástica. Videira-SC, 2010.

\begin{tabular}{ccccc}
\hline & & \multicolumn{3}{c}{ ETEFOM (g i.a. 100L $\left.\mathrm{L}^{-1}\right)$} \\
\cline { 2 - 5 } & & 0 & 136,8 & 273,6 \\
\hline \multirow{2}{*}{ ABA } & 0 & $537,6 \mathrm{bB}$ & $559,9 \mathrm{cB}$ & $601,5 \mathrm{cA}$ \\
(g i.a. $\left.100 \mathrm{~L}^{-1}\right)$ & 20 & $509,6 \mathrm{bB}$ & $626,3 \mathrm{bA}$ & $665,3 \mathrm{bA}$ \\
& 40 & $579,9 \mathrm{aB}$ & $687,4 \mathrm{aA}$ & $715,1 \mathrm{aA}$ \\
\hline
\end{tabular}

CV 5,94\%

Letras minúsculas iguais (na coluna) e maiúsculas iguais (na linha) não diferem ao nível de $5 \%$ de probabilidade de erro, pelo teste de Tukey. A unidade de medida de antocianinas é mg.L-1.

TABELA 4 - Efeito das aplicações de ABA e ETEFOM na concentração de polifenóis totais de cascas de uvas Cabernet Sauvignon sob cobertura plástica. Videira-SC, 2010.

\begin{tabular}{ccccc}
\hline & & \multicolumn{3}{c}{ ETEFOM (g i.a. 100L $\left.\mathrm{L}^{-1}\right)$} \\
\cline { 2 - 5 } & & 0 & 136,8 & 273,6 \\
\hline \multirow{2}{*}{ ABA } & 0 & $443,8 \mathrm{aA}$ & $460,0 \mathrm{bA}$ & $471,1 \mathrm{cA}$ \\
$\left(\right.$ g i.a. $\left.100 \mathrm{~L}^{-1}\right)$ & 20 & $417,4 \mathrm{aC}$ & $488,7 \mathrm{abB}$ & $560,9 \mathrm{bA}$ \\
& 40 & $454,0 \mathrm{aC}$ & $531,7 \mathrm{aB}$ & $619,7 \mathrm{aA}$ \\
\hline
\end{tabular}

Letras minúsculas iguais (na coluna) e maiúsculas iguais (na linha) não diferem ao nível de 5\% de probabilidade de erro, pelo teste de Tukey. A unidade de medida de polifenóis totais é mg. $\mathrm{L}^{-1}$.

TABELA 5 - Efeito das aplicações de ABA e ETEFOM na atividade antioxidante de cascas de uvas Cabernet Sauvignon sob cobertura plástica. Videira-SC, 2010.

\begin{tabular}{ccccc}
\hline & \multicolumn{3}{c}{ ETEFOM (g i.a. 100L $\left.\mathrm{L}^{-1}\right)$} \\
\cline { 2 - 5 } & & 0 & 136,8 & 273,6 \\
\hline ABA & 0 & $89,6 \mathrm{aA}$ & $90,7 \mathrm{aA}$ & $93,0 \mathrm{aA}$ \\
$\left(\right.$ g i.a. $\left.100 \mathrm{~L}^{-1}\right)$ & 20 & $91,3 \mathrm{aA}$ & $92,7 \mathrm{aA}$ & $94,0 \mathrm{aA}$ \\
& 40 & $92,3 \mathrm{aA}$ & $93,7 \mathrm{aA}$ & $92,7 \mathrm{aA}$ \\
\hline \multicolumn{4}{c}{ CV $1,92 \%$} \\
\hline
\end{tabular}

Letras minúsculas iguais (na coluna) e maiúsculas iguais (na linha) não diferem ao nível de $5 \%$ de probabilidade de erro, pelo teste de Tukey. A unidade de medida da atividade antioxidante é a porcentagem de sequestro de radicais livres DPPH. 
TABELA 6 - Efeito das aplicações de ABA e ETEFOM no conteúdo de sólidos solúveis totais ( ${ }^{\circ}$ Brix) de bagas de uvas Cabernet Sauvignon sob cobertura plástica. Videira-SC, 2010.

\begin{tabular}{ccccc}
\hline & & \multicolumn{3}{c}{ ETEFOM (g i.a. 100L $\mathrm{L}^{-1}$ ) } \\
\cline { 2 - 5 } & & 0 & 136,8 & 273,6 \\
\hline ABA & 0 & $19,52 \mathrm{bB}$ & $19,49 \mathrm{bB}$ & $20,38 \mathrm{aA}$ \\
$\left(\right.$ g i.a. $\left.100 \mathrm{~L}^{-1}\right)$ & 20 & $20,36 \mathrm{aB}$ & $20,88 \mathrm{aA}$ & $20,45 \mathrm{aAB}$ \\
& 40 & $20,33 \mathrm{aA}$ & $20,41 \mathrm{aA}$ & $20,48 \mathrm{aA}$ \\
\hline \multicolumn{4}{c}{ CV $1,12 \%$} \\
\hline
\end{tabular}

Letras minúsculas iguais (na coluna) e maiúsculas iguais (na linha) não diferem ao nível de $5 \%$ de probabilidade de erro, pelo teste de Tukey. A unidade de medida é ${ }^{\circ}$ Brix obtida com refratômetro de bancada.

TABELA 7 - Efeito das aplicações de ABA e ETEFOM na acidez total titulável de uvas Cabernet Sauvignon sob cobertura plástica. Videira-SC, 2010.

\begin{tabular}{ccccc}
\hline & & \multicolumn{3}{c}{ ETEFOM (g i.a. 100L-1) } \\
\cline { 2 - 5 } & & 0 & 136,8 & 273,6 \\
\hline \multirow{2}{*}{ ABA } & 0 & $7,28 \mathrm{bB}$ & $7,51 \mathrm{bA}$ & $6,75 \mathrm{cC}$ \\
(g i.a. 100L $\left.\mathrm{L}^{-1}\right)$ & 20 & $7,08 \mathrm{cB}$ & $7,20 \mathrm{cA}$ & $7,01 \mathrm{bB}$ \\
& 40 & $7,50 \mathrm{aB}$ & $7,69 \mathrm{aA}$ & $7,49 \mathrm{aB}$ \\
\hline \multicolumn{4}{c}{ CV 0,68\% } \\
\hline
\end{tabular}

Letras minúsculas iguais (na coluna) e maiúsculas iguais (na linha) não diferem ao nível de $5 \%$ de probabilidade de erro, pelo teste de Tukey. A unidade de medida é g.L $L^{-1}$ de ácido tartárico.

\section{CONCLUSÃO}

Neste contexto, pode-se concluir que o ABA melhora a pigmentação das cascas das uvas Cabernet Sauvignon. As quantidades de antocianinas e polifenóis nas uvas aumentaram com aplicações de ABA e ETEFOM. Por aumentam as concentrações de compostos fenólicos nas bagas, as aplicações aumentam a atividade antioxidante das uvas, e, por fim, o ABA e o ETEFOM alteram a maturação das uvas da cultivar Cabernet Sauvignon.

\section{REFERENCIAS}

ALMEIDA, J. M. et al. Avaliação da atividade antioxidante utilizando sistema B-caroteno/ácido linoleíco e método de sequestro de radicais DPPH. Ciência Tecnologia de Alimnetos, Campinas, v. 26, n. 2, p. 446-452, 2006.

CANTÍN, C. L.; FIDELIBUS, M. W.; CRISOSTO, C. H. Application of abscisic acid (ABA) at veraison advanced red color development and maintained postharvest quality of 'Crimson Seedless' grapes. Postharvest Biology and Technology, Amsterdam, v.46, n.3, p.237-241, 2007.
CAREÑO, J.; MARTINEZ, A.; ALMELA, L.; FERNANDEZ-LOPEZ, J. A. Proposal of an index for the objective evaluation of the color of red table grapes. Food Research International, Toronto, v.28, n.4, p.373-377, 1995.

COLLI, P.; PURGATT, E. Etileno. In: KERBAUY, G. B. Fisiologia vegetal. 2.ed. Rio de Janeiro: Guanabara, 2008. p.271-295.

DELGADO, R.; GALLEGOS, J. I.; MARTÍN, P.; GONZÁLEZ, M. R. Influence of ABA and Ethephon treatments on fruit composition of 'Trempranillo' grapevines. Acta Horticulturae, Wageningen, n.640, p.321-326, 2004.

EL KEREAMY, A.; CHERVIN, C.; ROUSTAN, J. P.; CHEYNIER, V.; SOUQUET, J. M.; MOUTOUMET, M.; RAYNAL, J.; FORD, C. M.; LATCHÉ, A.; PECH, J. C.; BOUZAYEN, M. Exogenous ethylene stimulates the long-term expression of genes related to anthocyanin biosynthesis in grape berries. Physiologia Plantarum, Copenhagem, v.119, n.2, p.175-182, 2003. 
GARDIN, J. P. P.; BETTONI, J.C.; TELES, O. R.; SCHUMACHER, R.L. Qualidade do mosto e exportação de nutrientes na cultivar Cabernet Sauvignon (Vitis vinifera 1.) em dois sistemas de cultivo. In: CONGRESSO BRASILEIRO DE FRUTICULTURA, 2010, Natal. Anais... São Paulo:SBF, 2010.

HIRATSUKA, S.; ONODERA, H.; KAWAI, Y.; KUBO, T.; ITOH, H.; WADA, R. ABA and sugar effects on anthocyanin formation in grape berry cultured in vitro. Scientia Horticulturae, Amsterdam, v.90, n.1-2, p.121-130, 2001.

KOLB, C.A.; KOPECKÝ; RIEDERER, M.; PFÜNDEL, E. UV screening by phenolics in berries of grapevine (Vitis vinifera). Functional Plant Biology, Victoria, n.30, v.12, p.1177-1186, 2003.

LACAMPAGNE, S.; GAGNÉ, S.; GÉNY, L. Involvement of Abscisic Acid in Controlling the Proanthocyanidin Biosynthesis Pathway in Grape Skin: New Elements Regarding the Regulation of Tannin Composition and Leucoanthocyanidin Reductase (LAR) and Anthocyanidin Reductase (ANR) Activities and Expression. Journal Plant Growth Regulation, New York, v.28, p.81-90, 2010.

PEPPI M. C.; FIDELIBUS, M. W.; DOKOOZLIAN, $\mathrm{N}$. Abscisic acid application timing and concentration affect firmness, pigmentation, and color of 'Flame Seedless' grapes. HortScience, Alexandria, v.41, p.1440-1445, 2006.

PEREIRA, F. M.; FRACARO, A. A. Efeito do ethephon na qualidade da uva 'niagara rosada' (Vitis labrusca L.), produzida na entressafra, na região de Jales-SP. Revista Brasileira de Fruticultura, Jaboticabal, v.26, n. 2, p.254-257, 2004.
R DEVELOPMENT CORE TEAM. R: A language and environment for statistical computing. Vienna: R Foundation for Statistical Computing, 2011. Disponível em: <http://www.R-project.org/>.

RIZZON, L. A. Metodologia para análise de mosto e suco de uva. Brasília: Embrapa Informação Tecnológica, 2010. 78p.

ROCHA, H. A.; GUERRA, N. B. Polifenóis em vinhos tintos: fatores envolvidos, propriedades funcionais e biodisponibilidade Revista Iberoamericana de Tecnología Postcosecha, México, v. 9, n.2, 2008, p.93-105.

RODRIGUES, A.; GIRARDI, E. A.; SCARPARE FILHO, J.A. Aplicação de Ethephon e qualidade da uva 'Rubi' em Porto Feliz-SP. 2010. Disponível em: <http://www.scielo.br/pdf/rbf/2010nahead/ aop11010.pdf $>$. Acesso em: 23 mar. 2012.

SOARES, M.; WELTER, L.; KUSKOSKI, E. M.; GONZAGA, L.; FETT, R. Compostos fenólicos e atividade antioxidante da casca de uvas niágara e Isabel. Revista Brasileira de Fruticultura, Jaboticabal, v. 30, n. 1, p.59-64, 2008

WATERHOUSE, A.L. Determination of total phenolics, in current protocols in food analytical chemistry. 2001. Disponível em: <http://www. nshtvn.org/ebook/molbio/Current\%20Protocols/ CPFAC/fai0101.pdf $>$. Acesso em: 11 jan. 2010. 